\title{
Spontaneous emission of a moving atom in the presence of magnetodielectric material: A relativistic approach
}

\author{
Fariba Shafieiyan $^{1}$, Ehsan Amooghorban ${ }^{11,2,3}$, and Ali Mahdifar ${ }^{1,2,4}$ \\ ${ }^{1}$ Department of Physics, Faculty of Basic Sciences, Shahrekord University, P.O. Box \\ 115, Shahrekord 88186-34141, Iran. \\ 2 Photonic Research Group, Shahrekord University, Shahrekord 88186-34141, Iran. \\ ${ }^{3}$ Nanotechnology Research Center, Shahrekord University, Shahrekord 88186-34141, \\ Iran. \\ ${ }^{4}$ Department of Physics, Faculty of Science, University of Isfahan, Hezar Jerib, Isfahan \\ 81746-73441, Iran.
}

\begin{abstract}
In this paper, based on a canonical quantization scheme, we study the effect of the relativistic motion of an excited atom on its decay rate in the presence of absorbing and dispersive media. For this purpose, we introduce an appropriate Lagrangian and describe the center-of-mass dynamical variables by the Dirac field. We obtain the Hamiltonian of the system in a multipolar form and calculate the motion equations of the system in the Schrödinger picture. We find that the decay rate and the quantum electrodynamics level shift of the moving atom can be expressed in terms of the imaginary part of the classical Green tensor and the center-of-mass velocity of the atom.
\end{abstract}

Keywords: Canonical Quantization, Spontaneous emission, Multipolar Hamiltonian, Röntgen interaction

PACS: 31.30.Jv, 03.70.+k,42.50.Nn,42.50.-p

\section{Introduction}

One of the most fundamental phenomena in quantum optics is the spontaneous emission caused by the inevitable interaction of an excited atom

\footnotetext{
${ }^{1}$ Ehsan.amooghorban@sci.sku.ac.ir
} 
with the vacuum-quantized electromagnetic field and/or the reaction of the atom to its own radiation field [1]. This process was first formulated theoretically by Dirac in 1927 and further by Weiskopff in 1930 [1]. In order to study the spontaneous emission, usually the atom is assumed to be in rest, which leads to difficulties due to Heisenberg's uncertainty relation [2]. In recent years there has been an increasing number of papers on the role of the center-of-mass motion in the process of spontaneous emission [3, 4, 5], Abraham-Minkowski-controversy [6, 7, 8, 9], Aharonov-Bohm-type phase shifts [10, 11, 12] and many important effects and applications associated with atomic motion in atom optics, laser cooling, trapping, and isotope separation experiments [13].

By taking into account the so-called Röntgen term in the atom-field interaction, Wilkens evaluated the velocity dependence of the spontaneous decay rate of an atom which moves in free space with a constant velocity $v$ to lowest order of $v / c$ [3]. Later, Boussiakou et al used a rigorous canonical formalism in which the center-of-mass dynamics of the atom is explicitly included and calculated the spontaneous decay of a moving excited atom in free space [4]. They showed that, irrespective of the orientation of the atomic dipole with respect to the direction of motion, the decay rate of the atom from the point of view of an observer in the laboratory frame is in agreement with special relativity. This result has been confirmed by an alternative but less general approach based on the basic principles of special relativity, physical processes associated with amoving electric dipole and the Doppler shift [5]. As a matter of fact, for more realistic cases atoms are not in free space, but move near the material media. Therefore, this is not a practical assumption for most real world applications.

It is well known that the presence of material media can change the structure of the fluctuating field of the vacuum. Consequently, the spontaneous emission rate can be modified if the atom moves with uniform nonrelativistic speed near materials of different composition and shape. In Refs. [14, 15, 16], authors considered a more realistic case and studied the spontaneous emission and the friction force experienced by an atom moving with uniform nonrelativistic velocity parallel to a dielectric surface. However, the question that naturally arises in this context is on the emission process occurring when an atom moves in absorbing magnetodielectric material relativistically. It is expected that the relativistic motion of the atom affects the radiative properties of the atom. The present paper is intended to respond this question. Our work extends previous works on the spontaneous decay of the moving 
excited atom in free space [4, 5], to the relativistic motion in the presence of the material media.

As a first step in studying the relativistic dynamics of a moving atom in the presence of absorbing media one has to provide the quantization of the electromagnetic field. Generally speaking, there are two approaches to quantize the electromagnetic field in the presence of material media: phenomenological and canonical approach. In this paper, we follow the canonical methods as presented in [17, 18, 19]. More details concerning this rigorous canonical approach can be found in references [17, 18, 19, 20, 21, 22, 23, 24].

The paper is organized as follows. In Sec. 2, we present a canonical quantization of the electromagnetic field interacting with moving charge particles in the presence of an isotropic, inhomogeneous and absorbing magnetodielectric medium. We start from a convenient Lagrangian and obtain the canonical momenta and the Hamiltonian of the combined system. We apply this approach to the case of two non-relativistic particles of opposite charges which form an atomic system. On the Hamiltonian, we perform a unitary transformation and derive nonrelativistic multipolar Hamiltonian in the electric-dipole approximation. In Sec. 3, we generalize the formalism by introducing the Dirac field to describe the relativistic motion of the atom. In Sec. 4, we examine the time evolution of the atomic system by treating the atom's external and internal degrees of freedom on the same quantum footing in the Schrödinger picture. In this section, the decay rate and the quantum electrodynamics level shift of the two-level atom, that moving with relativistic velocity near dissipative media, are explicitly evaluated. Finally, the main results are summarized in Sec. 5 .

\section{Basic equations for non-relativistic dynamics}

Let us consider a system composed of charged particles, the electromagnetic field, an absorbing and dispersive magnetodielectric medium and the interactions between them. Since, for short particle-medium separations the macroscopic description of the medium is not justified, we assume that the charged particles are placed in the free space and well separated from the medium. The Lagrangian of the whole system is written as follows [17, 18, 19, 20, 22, 23, 24]

$$
L=L_{q}+L_{e m}+L_{m}+L_{i n t},
$$


where

$$
L_{q}=\frac{1}{2} \sum_{\alpha} m_{\alpha} \dot{\mathbf{r}}_{\alpha}^{2}(t),
$$

is the Lagrangian for the charged particles with masses $m_{\alpha}$, charges $e_{\alpha}$ and the position vector $\mathbf{r}_{\alpha}$, and the Lagrangian of the electromagnetic field, $L_{e m}$, is given by $L_{e m}=\frac{1}{2} \int d^{3} \mathbf{r}\left(\varepsilon_{0} \mathcal{E}^{2}(\mathbf{r}, t)-\frac{\mathcal{B}^{2}(\mathbf{r}, t)}{\mu_{0}}\right)$. Here, the electric and magnetic fields can be defined in terms of the vector potential $\mathbf{A}$ and the scalar potential $\varphi$ as $\mathcal{E}=-\nabla \varphi-\frac{\partial \mathbf{A}}{\partial t}$ and $\mathcal{B}=\nabla \times \mathbf{A}$, respectively. In the Coulomb gauge, respectively, $-\nabla \varphi$ and $-\frac{\partial \mathbf{A}}{\partial t}$ are related to the longitudinal part $\mathcal{E}^{\|}$ and the transverse part $\mathcal{E}^{\perp}$ of the total electric field $\mathcal{E}$.

The third term in Eq. (1), $L_{m}$, denotes the magnetodielectric medium part as

$$
L_{m}=\frac{1}{2} \int d^{3} \mathbf{r} \int_{0}^{\infty} d \omega\left[\dot{\mathbf{X}}_{\omega}^{2}(\mathbf{r}, t)+\dot{\mathbf{Y}}_{\omega}^{2}(\mathbf{r}, t)-\omega^{2}\left(\mathbf{X}_{\omega}^{2}(\mathbf{r}, t)+\mathbf{Y}_{\omega}^{2}(\mathbf{r}, t)\right)\right]
$$

Here, the medium is modeled by two independent sets of harmonic oscillators characterized by means of two medium fields $\mathbf{X}_{\omega}$ and $\mathbf{Y}_{\omega}$. This scheme is based on Hopfield's microscopic model [25], which provide the dissipation of the energy as well as the polarizability and the magnetizability characters of the medium.

Finally, the interaction part of the Lagrangian (11) is given by

$$
\begin{aligned}
L_{i n t}= & \sum_{\alpha}\left[e_{\alpha} \dot{\mathbf{r}}_{\alpha} \cdot \mathbf{A}\left(\mathbf{r}_{\alpha}, t\right)-e_{\alpha} \varphi\left(\mathbf{r}_{\alpha}, t\right)\right] \\
& +\int d^{3} \mathbf{r}(\mathbf{P}(\mathbf{r}, t) \cdot \mathcal{E}(\mathbf{r}, t)+\mathbf{M}(\mathbf{r}, t) \cdot \mathcal{B}(\mathbf{r}, t)),
\end{aligned}
$$

where the terms in the first line describe the interaction of charged particles with the electromagnetic field, and those in the second line represent the interaction between the electromagnetic field and the material fields with the polarization vector $\mathbf{P}$ and the magnetization vector $\mathbf{M}$, respectively. Polarization and magnetization vectors can be, respectively, expressed in terms of the electric coupling function, $g_{e}$, and the magnetic coupling function, $g_{m}$, as follows

$$
\begin{aligned}
\mathbf{P}(\mathbf{r}, t) & =\int_{0}^{\infty} d \omega g_{e}(\mathbf{r}, \omega) \mathbf{X}_{\omega}(\mathbf{r}, t), \\
\mathbf{M}(\mathbf{r}, t) & =\int_{0}^{\infty} d \omega g_{m}(\mathbf{r}, \omega) \mathbf{Y}_{\omega}(\mathbf{r}, t) .
\end{aligned}
$$


We will see later that the dielectric permeability and the magnetic permittivity of the medium can be naturally expressed in terms of these coupling functions. To simplify the calculations, without loss of generality, we assume that the medium is isotropic. Therefore, the coupling functions $g_{e}$ and $g_{m}$ are both scalars, but take on tensor forms when the medium is anisotropic [19].

From the Lagrangian density (1), the canonical conjugate momenta associated to each dynamical variables can be obtained as

$$
\begin{gathered}
\mathbf{p}_{\alpha}(t)=\frac{\partial L}{\partial \dot{\mathbf{r}}_{\alpha}}=m_{\alpha} \dot{\mathbf{r}}_{\alpha}+e_{\alpha} \mathbf{A}\left(\mathbf{r}_{\alpha}, t\right), \\
-\varepsilon_{0} \mathcal{E}^{\perp}(\mathbf{r}, t)=\frac{\delta L}{\delta \dot{\mathbf{A}}(\mathbf{r}, t)}=\varepsilon_{0} \dot{\mathbf{A}}(\mathbf{r}, t), \\
\mathbf{Q}_{\omega}(\mathbf{r}, t)=\frac{\delta L}{\delta \dot{\mathbf{X}}_{\omega}(\mathbf{r}, t)}=\dot{\mathbf{X}}_{\omega}(\mathbf{r}, t)+g_{e}(\mathbf{r}, \omega) \mathbf{A}(\mathbf{r}, t), \\
\boldsymbol{\Pi}_{\omega}(\mathbf{r}, t)=\frac{\delta L}{\delta \dot{\mathbf{Y}}_{\omega}(\mathbf{r}, t)}=\dot{\mathbf{Y}}_{\omega}(\mathbf{r}, t) .
\end{gathered}
$$

To describe the system quantum mechanically, we follow the standard canonical quantization procedure and impose between the variables and their canonical conjugates, which are now operators on the Hilbert space, the following commutation relations

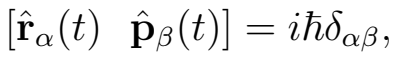

$$
\begin{aligned}
& {\left[\hat{\mathbf{A}}(\mathbf{r}, t)-\varepsilon_{0} \hat{\mathcal{E}}^{\perp}\left(\mathbf{r}^{\prime}, t\right)\right]=i \hbar \delta^{\perp}\left(\mathbf{r}-\mathbf{r}^{\prime}\right),} \\
& {\left[\hat{\mathbf{X}}_{\omega}(\mathbf{r}, t) \quad \hat{\mathbf{Q}}_{\omega^{\prime}}\left(\mathbf{r}^{\prime}, t\right)\right]=i \hbar \delta\left(\mathbf{r}-\mathbf{r}^{\prime}\right) \delta\left(\omega-\omega^{\prime}\right) \text {, }} \\
& {\left[\hat{\mathbf{Y}}_{\omega}(\mathbf{r}, t) \quad \hat{\boldsymbol{\Pi}}_{\omega^{\prime}}\left(\mathbf{r}^{\prime}, t\right)\right]=i \hbar \delta\left(\mathbf{r}-\mathbf{r}^{\prime}\right) \delta\left(\omega-\omega^{\prime}\right),}
\end{aligned}
$$

whereas all the other commutators of the canonical variables vanish. With the help of the above canonical momenta, we can now derive the hamiltonian 
of the system. After some algebra it reads

$$
\begin{aligned}
\hat{H} & =\sum_{\alpha} \frac{\left[\hat{\mathbf{p}}_{\alpha}-e_{\alpha} \hat{\mathbf{A}}\left(\hat{\mathbf{r}}_{\alpha}, t\right)\right]^{2}}{2 m_{\alpha}}+\frac{1}{2} \int d^{3} \mathbf{r}\left[\varepsilon_{0} \hat{\mathcal{E}}^{\perp 2}(\mathbf{r}, t)+\frac{\hat{\mathcal{B}}^{2}(\mathbf{r}, t)}{\mu_{0}}\right] \\
& +\frac{1}{2} \int d^{3} \mathbf{r} \int_{0}^{\infty} d \omega\left[\hat{\mathbf{Q}}_{\omega}^{2}(\mathbf{r}, t)+\omega^{2} \hat{\mathbf{X}}_{\omega}^{2}(\mathbf{r}, t)\right] \\
& +\frac{1}{2} \int d^{3} \mathbf{r} \int_{0}^{\infty} d \omega\left[\hat{\mathbf{\Pi}}_{\omega}^{2}(\mathbf{r}, t)+\omega^{2} \hat{\mathbf{Y}}_{\omega}^{2}(\mathbf{r}, t)\right] \\
& -\int d^{3} \mathbf{r}[\hat{\mathbf{M}}(\mathbf{r}, t) \cdot \hat{\mathcal{B}}(\mathbf{r}, t)+\hat{\mathbf{P}}(\mathbf{r}, t) \cdot \hat{\mathbf{A}}(\mathbf{r}, t)] \\
& -\frac{1}{2} \int d^{3} \mathbf{r} \int_{0}^{\infty} d \omega g_{e}(\mathbf{r}, \omega) \hat{\mathbf{A}}^{2}(\mathbf{r}, t)+w_{\text {coul }},
\end{aligned}
$$

where the Coulomb energy, $w_{\text {coul }}$, is due to the interactions between the charged particles, the charged particles and the polarization charges, and the interactions between the polarization charges, and is defined as follows [19]

$$
\begin{aligned}
w_{\text {coul }} & =\frac{1}{2} \int d^{3} \mathbf{r} \hat{\rho}_{A}(\mathbf{r}) \hat{\varphi}_{A}(\mathbf{r})+\int d^{3} \mathbf{r} \hat{\rho}_{A}(\mathbf{r}) \hat{\varphi}_{P}(\mathbf{r}) \\
& +\frac{1}{2} \int d^{3} \mathbf{r} \hat{\rho}_{P}(\mathbf{r}) \hat{\varphi}_{P}(\mathbf{r}) .
\end{aligned}
$$

Here, $\hat{\rho}_{A}(\mathbf{r})=\sum_{\alpha} e_{\alpha} \delta\left(\mathbf{r}-\hat{\mathbf{r}}_{\alpha}\right), \hat{\rho}_{P}(\mathbf{r})=-\nabla \cdot \hat{\mathbf{P}}(\mathbf{r})$, are, respectively, the charge density, the polarization charge density, and the scalar potential which are attributed to the external and the polarization charges, according to: $\hat{\varphi}_{A(P)}=\frac{1}{4 \pi \varepsilon_{0}} \int d^{3} \mathbf{r}^{\prime} \frac{\hat{\rho}_{A(P)}\left(\mathbf{r}^{\prime}\right)}{\left|\mathbf{r}-\mathbf{r}^{\prime}\right|}$. Due to the presence of the external charged particles, the longitudinal and transverse components of the electric field can be written respectively as $\hat{\mathcal{E}}^{\|}=\hat{\mathbf{E}}^{\|}-\nabla \hat{\phi}_{A}$ and $\hat{\mathcal{E}}^{\perp}=\hat{\mathbf{E}}^{\perp}$, wherein $\hat{\mathbf{E}}^{\|}=$ $-\nabla \hat{\varphi}_{P}=-\hat{\mathbf{P}}^{\|} / \varepsilon_{0}[19,26]$. Here, the total electric filed in the absence of the charged particles is denoted by $\hat{\mathbf{E}}$. Accordingly, the induction field $\hat{\mathbf{B}}$ in the presence and absence of the charged particles is given by $\hat{\mathcal{B}}=\hat{\mathbf{B}}[19]$.

In the Heisenberg picture, the time-evolution of operators are given by the Heisenberg equation. By using the Hamiltonian (15), the equations of motion for the material fields $\hat{\mathbf{X}}_{\omega}$ and $\hat{\mathbf{Y}}_{\omega}$ are obtained as

$$
\begin{aligned}
& \ddot{\hat{\mathbf{X}}}_{\omega}(\mathbf{r}, t)+\omega^{2} \hat{\mathbf{X}}_{\omega}(\mathbf{r}, t)=g_{e}(\mathbf{r}, \omega) \cdot \hat{\mathcal{E}}(\mathbf{r}, t), \\
& \ddot{\hat{\mathbf{Y}}}_{\omega}(\mathbf{r}, t)+\omega^{2} \hat{\mathbf{Y}}_{\omega}(\mathbf{r}, t)=g_{m}(\mathbf{r}, \omega) \cdot \hat{\mathbf{B}}(\mathbf{r}, t) .
\end{aligned}
$$


By solving these equations and substituting their solutions into Eqs. (5) and (6), we derive

$$
\begin{gathered}
\hat{\mathbf{P}}(\mathbf{r}, t)=\varepsilon_{0} \int_{0}^{\infty} d t^{\prime} \chi_{e}\left(\mathbf{r}, t-t^{\prime}\right) \hat{\mathcal{E}}(\mathbf{r}, t)+\hat{\mathbf{P}}^{N}(\mathbf{r}, t), \\
\hat{\mathbf{M}}(\mathbf{r}, t)=\mu_{0}^{-1} \int_{0}^{\infty} d t^{\prime} \chi_{m}\left(\mathbf{r}, t-t^{\prime}\right) \hat{\mathbf{B}}(\mathbf{r}, t)+\hat{\mathbf{M}}^{N}(\mathbf{r}, t) .
\end{gathered}
$$

where $\chi_{e}$ and $\chi_{m}$ are the electric and magnetic susceptibilities of the medium. In terms of the electric and magnetic coupling functions, $g_{e}$ and $g_{m}$, these expressions take the form

$$
\begin{aligned}
& \chi_{e}(\mathbf{r}, t)=\theta(t) \frac{1}{\varepsilon_{0}} \int_{0}^{\infty} d \omega g_{e}^{2}(\mathbf{r}, \omega) \frac{\sin \omega t}{\omega}, \\
& \chi_{m}(\mathbf{r}, t)=\theta(t) \mu_{0} \int_{0}^{\infty} d \omega g_{m}^{2}(\mathbf{r}, \omega) \frac{\sin \omega t}{\omega},
\end{aligned}
$$

where $\theta(t)$ is the Heaviside function.

In Eqs. (19) and (20), $\hat{\mathbf{P}}^{N}$ and $\hat{\mathbf{M}}^{N}$ are, respectively, the noise polarization and the noise magnetization operators. It is worth nothing that these noise operators form a Langevin noise current $\hat{\mathbf{J}}^{N}(\mathbf{r}, \omega)=-i \omega \hat{\mathbf{P}}^{N}(\mathbf{r}, \omega)+\nabla \times$ $\hat{\mathbf{M}}^{N}(\mathbf{r}, \omega)$ [27]. These operators can be expressed in terms of the homogeneous part of the solution of Eqs. (17) and (18) as follows:

$$
\begin{aligned}
\hat{\mathbf{P}}^{N}(\mathbf{r}, t) & =\int_{0}^{\infty} d \omega g_{e}(\mathbf{r}, \omega) \hat{\mathbf{X}}_{\omega}^{N}(\mathbf{r}, 0), \\
\hat{\mathbf{M}}^{N}(\mathbf{r}, t) & =\int_{0}^{\infty} d \omega g_{m}(\mathbf{r}, \omega) \hat{\mathbf{Y}}_{\omega}^{N}(\mathbf{r}, 0),
\end{aligned}
$$

where $\hat{\mathbf{X}}_{\omega}^{N}(\mathbf{r}, 0)=\dot{\hat{\mathbf{X}}}_{\omega}(\mathbf{r}, 0) \frac{\sin \omega t}{\omega}+\hat{\mathbf{X}}_{\omega}(\mathbf{r}, 0) \cos \omega t$ and $\hat{\mathbf{Y}}_{\omega}^{N}(\mathbf{r}, 0)=\dot{\hat{\mathbf{Y}}}_{\omega}(\mathbf{r}, 0) \frac{\sin \omega t}{\omega}+$ $\hat{\mathbf{Y}}_{\omega}(\mathbf{r}, 0) \cos \omega t$. The equation of motion for the variables of the electromagnetic field follows by deriving the Heisenberg equation for the electric field $\hat{\mathbf{E}}$. Using the solution of Eqs. (17) and (18) for the medium fields, one can easily show that the wave equation governing the electric field is obtained as

$$
\left[\nabla \times \mu^{-1}(\mathbf{r}, \omega) \nabla \times-\frac{\omega^{2}}{c^{2}} \varepsilon(\mathbf{r}, \omega)\right] \hat{\mathbf{E}}(\mathbf{r}, \omega)=i \omega \mu_{0} \hat{\mathbf{J}}^{N}(\mathbf{r}, \omega),
$$


where $c$ is the speed of light, and $\varepsilon=1+\chi_{e}$ and $\mu^{-1}=1-\chi_{m}$ are, respectively, the electric permittivity and the inverse magnetic permeability function of the medium.

Let us consider a simplified system of two non-relativistic particles of opposite charges $e_{1}=-e_{2}=e$ and masses $m_{1}$ and $m_{2}$ which form an atomic system, interacting with the electromagnetic field in the presence of a lossy medium. To separate the degrees of freedom due to the atomic gross motion from those of the internal motion, it is convenient to introduce the centerof-mass frame with new variables, $\hat{\mathbf{R}}=\sum_{\alpha=1}^{2} \frac{m_{\alpha} \hat{\mathbf{r}}_{\alpha}}{M}$ and $\hat{\mathbf{r}}_{r e l}=\hat{\mathbf{r}}_{1}-\hat{\mathbf{r}}_{2}$ with the corresponding conjugate momenta $\hat{\mathbf{\Pi}}\left(\hat{\mathbf{r}}_{\alpha}\right)=\hat{\mathbf{p}}_{1}\left(\hat{\mathbf{r}}_{1}\right)+\hat{\mathbf{p}}_{2}\left(\hat{\mathbf{r}}_{2}\right)$ and $\hat{\pi}\left(\hat{\mathbf{r}}_{\alpha}\right)=$ $\mu\left(\dot{\hat{\mathbf{r}}}_{1}-\dot{\hat{\mathbf{r}}}_{2}\right)$, where $M=m_{1}+m_{2}$ and $\mu=\frac{m_{1} m_{2}}{M}$ are the atomic and reduced mass, respectively. From the above definition of the new variables and making use of the commutation relations (11), it immediately follows that

$$
\begin{aligned}
& {\left[\hat{R}_{i}, \hat{\Pi}_{j}\right]=i \hbar \delta_{i j},} \\
& {\left[\hat{r}_{i}^{r e l}, \hat{\pi}_{j}\right]=i \hbar \delta_{i j} .}
\end{aligned}
$$

In the following, we rephrase the Hamiltonian (15) in terms of the center-ofmass dynamical variables. This can be carried out by applying a canonical transformation to Eq. (15) and, when explicitly written, is seen to lead to the usual multipolar Hamiltonian. The canonical transformation is nothing other than the Power-Zienau-Woolley transformation which is characterized by the unitary operator $\hat{U}=e^{i \hat{\Lambda}}=\exp \left[\frac{i}{\hbar} \int d^{3} \mathbf{r} \hat{\mathbf{P}}_{a t}(\mathbf{r}, t) \cdot \hat{\mathbf{A}}(\mathbf{r}, t)\right]$ where

$$
\hat{\mathbf{P}}_{a t}(\mathbf{r}, t)=\sum_{\alpha=1}^{2} e_{\alpha}\left(\hat{\mathbf{r}}_{\alpha}-\hat{\mathbf{R}}\right) \int_{0}^{1} d \sigma \delta\left(\mathbf{r}-\hat{\mathbf{R}}-\sigma\left(\hat{\mathbf{r}}_{\alpha}-\hat{\mathbf{R}}\right)\right) .
$$

is the atomic polarization field relative to the center-of-mass coordinate $\hat{\mathbf{R}}$ [29]. Under this canonical transformation, it is clearly seen that the variables $\hat{\mathbf{r}}_{\alpha}, \hat{\mathbf{A}}$, and $\hat{\mathbf{B}}$ are unchanged. In contrast, the canonical momentum $\hat{\mathbf{p}}_{\alpha}$ and the transverse part of the electric field $\hat{\mathbf{E}}^{\perp}$ are modified as follows [28, 29]

$$
\begin{aligned}
\hat{\mathbf{p}}_{\alpha}^{\prime}= & \hat{U}^{\dagger} \hat{\mathbf{p}}_{\alpha} \hat{U} \\
= & \hat{\mathbf{p}}_{\alpha}+e_{\alpha} \hat{\mathbf{A}}\left(\hat{\mathbf{r}}_{\alpha}\right)+\int d^{3} \mathbf{r} \hat{\Xi}_{a t, \alpha}(\mathbf{r}) \times \hat{\mathbf{B}}(\mathbf{r}), \\
\hat{\mathbf{E}}^{\prime \perp}(\mathbf{r}, t) & =\hat{U}^{\dagger} \hat{\mathbf{E}}^{\perp}(\mathbf{r}, t) \hat{U} \\
& =\hat{\mathbf{E}}^{\perp}(\mathbf{r}, t)-\frac{1}{\varepsilon_{0}} \hat{\mathbf{P}}_{a t}^{\perp}(\mathbf{r}, t),
\end{aligned}
$$


where $\nabla^{\alpha}$ refers to differentiation with respect to the coordinate $\hat{\mathbf{r}}_{\alpha}$, the subscript $\perp$ indicates the transverse part of the relevant vector, and $\hat{\boldsymbol{\Xi}}_{a t, \alpha}(\mathbf{r})$ is the atomic magnetization field relative to the center-of-mass coordinate $\hat{\mathbf{R}}$, which is defined as:

$\hat{\boldsymbol{\Xi}}_{a t, \alpha}(\mathbf{r})=\sum_{\beta=1}^{2} e_{\beta} \int_{0}^{1} d \sigma\left(\sigma \delta_{\alpha \beta}-\frac{m_{a}}{M}[\sigma-1]\right)\left[\hat{\mathbf{r}}_{\beta}-\hat{\mathbf{R}}\right] \delta\left(\mathbf{r}-\hat{\mathbf{R}}-\sigma\left(\hat{\mathbf{r}}_{\alpha}-\hat{\mathbf{R}}\right)\right)$.

Now, we obtain the multipolar Hamiltonian, $\hat{H}_{\text {mult }}=\hat{U}^{\dagger} \hat{H} \hat{U}$, in the electric dipole approximation. In order to get the electric dipole approximation of the multipolar Hamiltonian, we expand the Dirac $\delta$ distributions appearing in the atomic polarization vectors $\hat{\mathbf{P}}_{a t}$ and $\hat{\boldsymbol{\Xi}}_{\alpha}$ in powers of $\hat{\mathbf{r}}_{\alpha}-\hat{\mathbf{R}}$ and keeping only the leading terms. Using the electric dipole version of Eqs. (28) and (31), after some lengthy manipulations the multipolar Hamiltonian in the electric dipole approximation is obtained as:

$$
\begin{aligned}
\hat{H}_{\text {mult }} & =\frac{\hat{\boldsymbol{\Pi}}^{2}}{2 M}+\left(\frac{\hat{\mathbf{p}}^{2}}{2 \mu}-\frac{e^{2}}{4 \pi \varepsilon_{0} \hat{\mathbf{r}}_{r e l}}\right)+\frac{1}{2} \int d^{3} \mathbf{r}\left[\varepsilon_{0} \hat{\mathbf{E}}^{\perp 2}(\mathbf{r}, t)+\frac{\hat{\mathbf{B}}^{2}(\mathbf{r}, t)}{\mu_{0}}\right] \\
& +\frac{1}{2} \int d^{3} \mathbf{r} \int_{0}^{\infty} d \omega\left[\dot{\hat{\mathbf{X}}}_{\omega}^{2}(\mathbf{r}, t)+\dot{\hat{\mathbf{Y}}}_{\omega}^{2}(\mathbf{r}, t)+\omega^{2}\left(\hat{\mathbf{X}}_{\omega}^{2}(\mathbf{r}, t)+\hat{\mathbf{Y}}_{\omega}^{2}(\mathbf{r}, t)\right)\right] \\
& -\int d^{3} \mathbf{r} \hat{\mathbf{M}}(\mathbf{r}, t) \cdot \hat{\mathbf{B}}(\mathbf{r}, t)-\int d^{3} \mathbf{r} \hat{\mathbf{d}} \cdot \hat{\mathbf{E}}(\hat{\mathbf{R}}) \\
& +\frac{1}{2 M} \int d^{3} \mathbf{r}(\hat{\mathbf{\Pi}} \cdot \hat{\mathbf{d}} \times \hat{\mathbf{B}}(\hat{\mathbf{R}})+\hat{\mathbf{d}} \times \hat{\mathbf{B}}(\hat{\mathbf{R}}) \cdot \hat{\mathbf{\Pi}})+\frac{\hat{\mathbf{d}} \times \hat{\mathbf{B}}(\mathbf{R})}{8 \mu} \\
& +\frac{1}{2 \varepsilon_{0}} \int d^{3} \mathbf{r} \hat{\mathbf{P}}_{a t}^{\perp}+w_{\text {coul }}+(\text { magnetic }- \text { dipoleterm }),
\end{aligned}
$$

where $\hat{\mathbf{d}}=e \hat{\mathbf{r}}_{r e l}$ is the atomic transition dipole momentum in the laboratory frame with matrix elements $\mathbf{d}_{k l}=\langle k|\hat{\mathbf{d}}| l\rangle$, the $\hat{\boldsymbol{\Pi}}$-dependent terms refer to the Röntgen interaction which couples the electric moment of the atom to the magnetic field by virtue of the motion of the atom, the magnetic dipole term comes from the product between $\hat{\pi}$ and $\hat{\mathbf{d}} \times \hat{\mathbf{B}}(\hat{\mathbf{R}})$ and would be negligible compared to the electric dipole interaction [28], the term $\frac{[\hat{\mathbf{d}} \times \hat{\mathbf{B}}(\hat{\mathbf{R}})]}{8 \mu}$ represents the diamagnetic interaction energy and is small enough to ignore in our calculations [30], and the term involving the integral of the square of the atomic polarization contributes to the Lamb shift and may be omitted in renormalized energies of the internal motion. 
Let us model the atom as a two-level atom with the ground state $|l\rangle$, the excited state $|u\rangle$ and the energy difference $\hbar \omega_{A}$. With above points in view and introducing the explicit information about the energy levels to Eq. (32), we arrive at the following effective Hamiltonian

$$
\begin{aligned}
\hat{H}_{m u l t}^{\text {eff }} & =\frac{\hat{\boldsymbol{\Pi}}^{2}}{2 M}+\hbar \omega_{A} \hat{\sigma}^{\dagger} \hat{\sigma}+\frac{1}{2} \int d^{3} \mathbf{r}\left(\varepsilon_{0} \hat{\mathbf{E}}^{\perp 2}(\mathbf{r}, t)+\frac{\hat{\mathbf{B}}^{2}(\mathbf{r}, t)}{\mu_{0}}\right) \\
& +\frac{1}{2} \int d^{3} \mathbf{r} \int_{0}^{\infty} d \omega\left[\dot{\hat{\mathbf{X}}}_{\omega}^{2}(\mathbf{r}, t)+\dot{\hat{\mathbf{Y}}}_{\omega}^{2}(\mathbf{r}, t)+\omega^{2}\left(\hat{\mathbf{X}}_{\omega}^{2}(\mathbf{r}, t)+\hat{\mathbf{Y}}_{\omega}^{2}(\mathbf{r}, t)\right)\right] \\
& -\int d^{3} \mathbf{r} \hat{\mathbf{M}}(\mathbf{r}, t) \cdot \hat{\mathbf{B}}(\mathbf{r}, t)-\int d^{3} \mathbf{r} \hat{\mathbf{d}} \cdot \hat{\mathbf{E}}(\hat{\mathbf{R}}) \\
& +\frac{1}{2 M} \int d^{3} \mathbf{r}(\hat{\mathbf{\Pi}} \cdot \hat{\mathbf{d}} \times \hat{\mathbf{B}}(\hat{\mathbf{R}})+\hat{\mathbf{d}} \times \hat{\mathbf{B}}(\hat{\mathbf{R}}) \cdot \hat{\mathbf{\Pi}})
\end{aligned}
$$

where $\hat{\sigma}$ and $\hat{\sigma}^{\dagger}$ are the Pauli lowering and raising operators of the two-level atom.

To facilitate calculations, we introduce the bosonic operators $\hat{\mathbf{f}}_{e}(\mathbf{r}, \omega, t)=$ $\frac{1}{\sqrt{2 \hbar \omega}}\left[-i \omega \hat{\mathbf{X}}_{\omega}(\mathbf{r}, t)+\hat{\mathbf{Q}}_{\omega}(\mathbf{r}, t)\right]$ and $\hat{\mathbf{f}}_{m}(\mathbf{r}, \omega, t)=\frac{1}{\sqrt{2 \hbar \omega}}\left[\omega \hat{\mathbf{Y}}_{\omega}(\mathbf{r}, t)+i \hat{\mathbf{\Pi}}_{\omega}(\mathbf{r}, t)\right]$ which play the roll of the collective excitations of the electromagnetic field and the absorbing medium. By lengthy but straightforward calculations, one can show that the Hamiltonian of the electromagnetic field, the medium and the interactions between them, i.e. the last term in the first line, the second line and the first term in the third line of Eq. (33), are simplified as [17, 20, 24]

$$
\hat{H}_{F}=\sum_{\lambda=e, m} \int d \omega \int d^{3} \mathbf{r} \hbar \omega \hat{\mathbf{f}}_{\lambda}^{\dagger}(\mathbf{r}, \omega, t) \cdot \hat{\mathbf{f}}_{\lambda}(\mathbf{r}, \omega, t)
$$

where $\hat{\mathbf{f}}_{e}(\mathbf{r}, \omega, t)$ and $\hat{\mathbf{f}}_{m}(\mathbf{r}, \omega, t)$ associated with the electric and magnetic excitations. By applying the commutation relations (13) and (14), it can be shown that the bosonic operators satisfy the usual commutation relations

$$
\left[\hat{f}_{\lambda, j}(\mathbf{r}, \omega, t), \hat{f}_{\lambda^{\prime}, j^{\prime}}^{\dagger}\left(\mathbf{r}^{\prime}, \omega^{\prime}, t\right)\right]=\delta_{j j^{\prime}} \delta\left(\mathbf{r}-\mathbf{r}^{\prime}\right) \delta\left(\omega-\omega^{\prime}\right) .
$$

With the help of these bosonic operators and Eqs. (25), (23) and (24), the explicit form of the electric field operator is obtained in terms of the electro- 
magnetic Green tensor of the system as follows:

$$
\begin{aligned}
\hat{\mathbf{E}}(\mathbf{r}, t) & =i \sqrt{\frac{\hbar \mu_{0}}{\pi}} \int d^{3} \mathbf{r}^{\prime} \int_{0}^{\infty} d \omega \omega \frac{\omega}{c} \sqrt{\operatorname{Im} \varepsilon\left(\mathbf{r}^{\prime}, \omega\right)} \mathbf{G}\left(\mathbf{r}, \mathbf{r}^{\prime}, \omega\right) \cdot \hat{\mathbf{f}}_{e}\left(\mathbf{r}^{\prime}, \omega\right) \\
& +\sqrt{\frac{\operatorname{Im} \mu\left(\mathbf{r}^{\prime}, \omega\right)}{\left|\mu\left(\mathbf{r}^{\prime}, \omega\right)\right|^{2}}}\left[\nabla^{\prime} \times \mathbf{G}\left(\mathbf{r}^{\prime}, \mathbf{r}, \omega\right)\right]^{T} \cdot \hat{\mathbf{f}}_{m}\left(\mathbf{r}^{\prime}, \omega\right)+H \cdot C \cdot,
\end{aligned}
$$

where $\mathbf{G}\left(\mathbf{r}, \mathbf{r}^{\prime}, \omega\right)$ is the unique solution to the Helmholtz equation

$$
\left[\nabla \times \mu^{-1}(\mathbf{r}, \omega) \nabla \times-\frac{\omega^{2}}{c^{2}} \varepsilon(\mathbf{r}, \omega)\right] \mathbf{G}\left(\mathbf{r}, \mathbf{r}^{\prime}, \omega\right)=\delta\left(\mathbf{r}-\mathbf{r}^{\prime}\right) \overline{\bar{I}}
$$

together with the boundary condition $\mathbf{G}\left(\mathbf{r}, \mathbf{r}^{\prime}, \omega\right) \rightarrow 0$ for $\left|\mathbf{r}-\mathbf{r}^{\prime}\right| \rightarrow \infty$, satisfies the integral relation

$$
\begin{aligned}
& \int d^{3} \mathbf{s}\left(-\operatorname{Im}\left[\mu^{-1}(\mathbf{s}, \omega)\right]\left[\nabla_{\mathbf{s}} \times \mathbf{G}(\mathbf{s}, \mathbf{r}, \omega)\right]^{T} \cdot\left[\nabla_{\mathbf{s}} \times \mathbf{G}^{*}\left(\mathbf{s}, \mathbf{r}^{\prime}, \omega\right)\right]\right. \\
& \left.+\frac{\omega^{2}}{c^{2}} \operatorname{Im}[\varepsilon(\mathbf{s}, \omega)] \mathbf{G}(\mathbf{r}, \mathbf{s}, \omega) \cdot \mathbf{G}^{*}\left(\mathbf{s}, \mathbf{r}^{\prime}, \omega\right)\right)=\operatorname{Im}\left[\mathbf{G}\left(\mathbf{r}, \mathbf{r}^{\prime}, \omega\right)\right] .
\end{aligned}
$$

All the information about the geometry and topology of the environmental media are contained in the Green tensor of the system $\mathbf{G}$ via the electric permittivity function $\varepsilon$ and the magnetic permeability function $\mu$. Now, the set of equations (33), (34) and (36) together with the commutation relations (26), (27) and (35) provide the canonical quantization of the electromagnetic field interacting with moving charge particles in the presence of an isotropic, inhomogeneous and absorbing magnetodielectric medium. As we expected, these equations are the same as obtained from the phenomenological method [31, 32]. In recent years, based on the phenomenological approach, the quantum description of moving atoms at non-relativistic speeds has been studied extensively [14, 31, 32]. In the next section, as an application of our formalism and a main purpose of this article pointed out earlier, we treat the general case that the atomic system is in uniform motion with relativistic speeds in the presence of material medium.

\section{Relativistic dynamics}

In this section, we study the dynamics of a moving two-level atom interacting with the electromagnetic field near absorbing media at the relativistic 
regime. The starting point is the total Lagrangian (1), with the only difference is that the particle motion is treated relativistically. Because the medium is at rest, therefore, there is no need to write the Lagrangian density associated with the medium in a covariant form. Given that the internal dynamics of the atom are not affected by relativistic considerations other than through $\hat{\mathbf{R}}$ [4], we therefore follow the approach presented in [17, 33] and describe the center-of-mass dynamical variables by the Dirac field. To do so, we start from the total Lagrangian (1), and rewrite the Lagrangian associated with the kinetic energy of the charged particles and their interactions with the electromagnetic field in term of the center-of-mass coordinate $\hat{\mathbf{R}}$ and the relative coordinate $\hat{\mathbf{r}}_{\text {rel }}$. With these notes, we can replace the relevant terms associated to the center-of-mass coordinate by

$$
L_{D}=\frac{i \hbar c}{2} \int d^{3} \mathbf{r}\left\{\sum_{\mu=1}^{4}\left(\bar{\psi}(\mathbf{r}, t) \gamma^{\mu} \frac{\partial \psi(\mathbf{r}, t)}{\partial \mathrm{r}^{\mu}}-\frac{\partial \bar{\psi}(\mathbf{r}, t)}{\partial \mathrm{r}^{\mu}} \gamma^{\mu} \psi(\mathbf{r}, t)\right)-M c^{2} \bar{\psi} \psi\right\}
$$

and write the external current and charge densities, respectively, as $\mathbf{J}(\mathbf{r}, t)=$ $e c \psi^{\dagger}(\mathbf{r}, t) \boldsymbol{\alpha} \psi(\mathbf{r}, t)$ and $\rho(\mathbf{r}, t)=e \psi^{\dagger} \psi(\mathbf{r}, t)$, where $\gamma^{\mu}$ are the Dirac matrices with $\gamma^{4}=\beta, \gamma^{j}=\beta \alpha_{j}$, and $\bar{\psi}=\psi^{\dagger} \beta$. In the standard representation, the $\gamma$ matrices are expressed in terms of the $2 \times 2$ Pauli matrices $\sigma_{j}(j=1,2,3)$ and the identity matrix $\mathbb{I}$, as

$$
\beta=\left(\begin{array}{cc}
\mathbb{I} & 0 \\
0 & -\mathbb{I}
\end{array}\right), \quad \alpha_{j}=\left(\begin{array}{cc}
0 & \sigma_{j} \\
\sigma_{j} & 0
\end{array}\right)
$$

The canonical momentum associated to $\psi$ is given by $i \hbar \psi^{\dagger} / 2=\frac{\partial L}{\partial \dot{\psi}(\mathbf{r}, t)}$. Now, we impose the canonical anti-commutation relation for the Dirac field as

$$
\begin{gathered}
\left\{\psi_{\alpha}(\mathbf{r}, t), \psi_{\beta}^{\dagger}\left(\mathbf{r}^{\prime}, t\right)\right\}=\delta_{\alpha \beta} \delta\left(\mathbf{r}-\mathbf{r}^{\prime}\right), \\
\left\{\psi_{\alpha}(\mathbf{r}, t), \psi_{\beta}\left(\mathbf{r}^{\prime}, t\right)\right\}=0
\end{gathered}
$$

It is convenient to expand the Dirac field $\psi(\mathbf{r}, t)$ in terms of the eigenfunctions of Dirac equation in the absence of electromagnetic field

$$
\psi(\mathbf{r}, t)=\frac{1}{(2 \pi)^{3 / 2}} \sum_{\mu=1}^{4} \int d^{3} \mathbf{q} \hat{c}_{\mu}(\mathbf{q}, t) \psi_{\mu}(\mathbf{q}),
$$


where $\psi_{\mu}(\mathbf{q})=u_{\mu}(\mathbf{q}) e^{i \mathbf{q} \cdot \mathbf{r}}$ with $u_{\mu}(\mathbf{q})$ are the four-component spinors and $\hat{c}_{\mu}(\mathbf{q}, t)$ is the atomic annihilation operator [34]. With the help of the above expansion and by substituting Eq. (39) into (11) and making use of Eqs. (8)(10) and the canonical momentum $\psi^{\dagger}$, we obtain the effective multipolar Hamiltonian as

$$
\hat{H}_{\text {mult }}=\hat{H}_{A}+\hat{H}_{F}+\hat{H}_{\text {int }},
$$

where $\hat{H}_{F}$ is the Hamiltonian given by Eq. (34) and $\hat{H}_{A}=\frac{1}{2} \frac{\hbar \omega_{A}}{\gamma} \hat{\sigma}^{\dagger} \hat{\sigma}+$ $\sum_{\mu=1}^{4} \int d^{3} \mathbf{q} E_{\mathbf{q}, \mu} \hat{c}_{\mu}^{\dagger}(\mathbf{q}, t) \hat{c}_{\mu}(\mathbf{q}, t)$ denotes the Hamiltonian of the moving atom, wherein $\gamma=\sqrt{1-\dot{\mathbf{R}}^{2} / c^{2}}$ with $\dot{\mathbf{R}}$ is the center-of-mass velocity of the atom. Here, the first term in the right-hand side of $\hat{H}_{A}$ corresponds to the internal dynamics of the atom in the laboratory frame, while the second term is related to the center-of-mass motion of the atom with the momentum $\hbar \mathbf{q}$ and the energy $E_{\mathbf{q}, \mu}= \pm \sqrt{\hbar^{2} c^{2} q^{2}+M^{2} c^{4}}$, in which the plus(minus) sign is to be taken for $\mu=1,2(\mu=3,4)$. The unperturbed Hamiltonian $\hat{H}_{A}+\hat{H}_{F}$ has the eigenstate $\mid$ atom $+\mathrm{rad}\rangle=\mid$ atom $\rangle|\mathrm{rad}\rangle$ which are the direct product of the eigenstates of $\hat{H}_{A}$ and $\hat{H}_{F}$.

The interaction Hamiltonian $\hat{H}_{\text {int }}$ in Eq. (44) composed of two parts: the interaction between the electric dipole and the electric and magnetic field, and the Röntgen interaction. The interaction Hamiltonian in the Fourier space is given by

$$
\begin{aligned}
& \hat{H}_{\text {int }}^{\text {eff }}=-\frac{i \mu_{0}}{(2 \pi)^{3}} \hat{\sigma}^{\dagger} \hat{\mathbf{d}}_{A} \cdot \sum_{\mu, \mu^{\prime}=1}^{4} \int_{0}^{\infty} d \omega \omega \int d^{3} \mathbf{q} \int d^{3} \mathbf{k} \int d^{3} \mathbf{k}_{1} \int d^{3} \mathbf{k}_{2} \\
& \times \mathbf{G}\left(\mathbf{k}_{2}, \mathbf{k}, \omega\right)\left[\omega g_{e}\left(\mathbf{k}-\mathbf{k}_{1}, \omega\right) \cdot \hat{\mathbf{f}}_{e}\left(\mathbf{k}_{1}, \omega\right)+i \mathbf{k} g_{m}\left(\mathbf{k}-\mathbf{k}_{1}, \omega\right) \cdot \hat{\mathbf{f}}_{m}\left(\mathbf{k}_{1}, \omega\right)\right] \\
& \times u_{\mu}^{\dagger}(\mathbf{q}) u_{\mu^{\prime}}\left(\mathbf{q}-\mathbf{k}_{2}\right) \hat{c}_{\mu}^{\dagger}(\mathbf{q}, t) \hat{c}_{\mu^{\prime}}\left(\mathbf{q}-\mathbf{k}_{2}, \omega\right)+H \cdot C \cdot \\
& -\frac{i \mu_{0}}{(2 \pi)^{3}} \hat{\sigma}^{\dagger} \mathbf{d}_{A} \cdot \sum_{\mu, \mu^{\prime}=1}^{4} \int_{0}^{\infty} d \omega \int d^{3} \mathbf{q} \int d^{3} \mathbf{k} \int d^{3} \mathbf{k}_{1} \int d^{3} \mathbf{k}_{2} \\
& \times \dot{\mathbf{R}} \times \mathbf{k}_{2} \times \mathbf{G}\left(\mathbf{k}_{2}, \mathbf{k}, \omega\right)\left[\omega g_{e}\left(\mathbf{k}-\mathbf{k}_{1}, \omega\right) \cdot \hat{\mathbf{f}}_{e}\left(\mathbf{k}_{1}, \omega\right)\right. \\
& \left.+i \mathbf{k} g_{m}\left(\mathbf{k}-\mathbf{k}_{1}, \omega\right) \cdot \hat{\mathbf{f}}_{m}\left(\mathbf{k}_{1}, \omega\right)\right] \\
& \times u_{\mu}^{\dagger}(\mathbf{q}) u_{\mu^{\prime}}\left(\mathbf{q}-\mathbf{k}_{2}\right) \hat{c}_{\mu}^{\dagger}(\mathbf{q}, t) \hat{c}_{\mu^{\prime}}\left(\mathbf{q}-\mathbf{k}_{2}, \omega\right)+H \cdot C \cdot,
\end{aligned}
$$

where $\mathbf{d}_{A}=\langle l|\hat{\mathbf{d}}| u\rangle=\langle u|\hat{\mathbf{d}}| l\rangle$. 


\section{Radiative properties of a moving atom}

In this section, to describe the radiative dynamics of the moving atom, we study the time evolution of the system state vector and find their probability amplitudes by using the Markov approximation. For a single-quantum excitation, the time-dependent state vector of the whole system can be written as

$$
\begin{aligned}
|\psi(\mathbf{q}, t)\rangle= & \sum_{\mu=1}^{2} \int d^{3} \mathbf{q} C_{u, \mu}(\mathbf{q}, t) e^{-i \tilde{\omega}_{A} t}|\mathbf{q}\rangle_{\mu}|u\rangle|\{0\}\rangle \\
+ & \sum_{\lambda=e, m} \sum_{\mu=1}^{2} \int d^{3} \mathbf{q} \int d^{3} \mathbf{k} \int d^{3} \mathbf{k}^{\prime} \int d \omega e^{-i\left(\omega+\omega_{B}\right) t} \\
& \times C_{\lambda l, \mu}\left(\mathbf{q}, \mathbf{k}, \mathbf{k}^{\prime}, \omega, t\right)\left|\mathbf{q}-\mathbf{k}^{\prime}\right\rangle_{\mu}|l\rangle\left|\left\{\mathbf{1}_{\lambda}\left(\mathbf{k}^{\prime}, \omega\right)\right\}\right\rangle .
\end{aligned}
$$

where the atomic state vectors $|\mathbf{q}\rangle_{\mu}$ and $\left|\mathbf{q}-\mathbf{k}^{\prime}\right\rangle_{\mu}$ refer to the momentum of the atom before and after the emission of the photon, $|\{0\}\rangle$ is the vacuum state of the field, $\left|\left\{\mathbf{1}_{\lambda}(\mathbf{k}, \omega)\right\}\right\rangle$ is the excited state of the field with $\hat{\mathbf{f}}_{\lambda}^{\dagger}(\mathbf{k}, \omega)|\{0\}\rangle=\left|\left\{\mathbf{1}_{\lambda}(\mathbf{k}, \omega)\right\}\right\rangle$, and $C_{u, \mu}(\mathbf{q}, t)$ and $C_{\lambda l, \mu}\left(\mathbf{q}, \mathbf{k}, \mathbf{k}^{\prime}, \omega, t\right)$ are the respective probability amplitudes of the excited and ground states of the system, the subscript $\lambda=e, m$ indicates the electric and magnetic excitations of the medium, and the frequencies $\tilde{\omega}_{A}$ and $\omega_{B}$ are, respectively, defined as $\tilde{\omega}_{A}=\frac{\omega_{A}}{\gamma}+\frac{\left|E_{\mathbf{q}}\right|}{\hbar}-\delta \omega$ and $\omega_{B}=\frac{\left|E_{\mathbf{q}-\mathbf{k}^{\prime}}\right|}{\hbar}$ with $\delta \omega$ denoting the Lamb shift. In this case the dynamics is described by the time-dependent Schrödinger equation $i \hbar \partial_{t}|\psi(t)\rangle=\hat{H}|\psi(t)\rangle$. We insert the state vector (46) into the 
Schrödinger equation, and obtain the following equations of motion:

$$
\begin{aligned}
& \dot{C}_{u, \mu}(\mathbf{q}, t)=-i \delta \omega C_{u, \mu}(\mathbf{q}, t) \\
& -\frac{1}{(2 \pi)^{3} c \sqrt{\pi \varepsilon_{0} \hbar}} \sum_{\mu^{\prime}=1}^{2} \int_{0}^{\infty} d \omega e^{-i\left(\omega+\omega_{B}-\tilde{\omega}_{A}\right) t} \int d^{3} \mathbf{k} \int d^{3} \mathbf{k}^{\prime} \int d^{3} \mathbf{k}_{1} \\
& \times \hat{\mathbf{d}}_{A} \cdot\left\{\left[\frac{\omega^{2}}{c} g_{e}\left(\mathbf{k}_{1}-\mathbf{k}, \omega\right) \mathbf{G}\left(\mathbf{k}^{\prime}, \mathbf{k}_{1}, \omega\right) C_{e l, \mu^{\prime}}\left(\mathbf{q}, \mathbf{k}, \mathbf{k}^{\prime}, \omega, t\right)\right.\right. \\
& \left.+i \omega g_{m}\left(\mathbf{k}_{1}-\mathbf{k}, \omega\right) \mathbf{G}\left(\mathbf{k}^{\prime}, \mathbf{k}_{1}, \omega\right) \times \mathbf{k}_{1} C_{m l, \mu^{\prime}}\left(\mathbf{q}, \mathbf{k}, \mathbf{k}^{\prime}, \omega, t\right)\right] \\
& +\dot{\mathbf{R}} \times \mathbf{k}^{\prime} \times\left[\frac{\omega}{c} g_{e}\left(\mathbf{k}_{1}-\mathbf{k}, \omega\right) \mathbf{G}\left(\mathbf{k}^{\prime}, \mathbf{k}_{1}, \omega\right) C_{e l, \mu^{\prime}}\left(\mathbf{q}, \mathbf{k}, \mathbf{k}^{\prime}, \omega, t\right)\right. \\
& \left.\left.+i g_{m}\left(\mathbf{k}_{1}-\mathbf{k}, \omega\right) \mathbf{G}\left(\mathbf{k}^{\prime}, \mathbf{k}_{1}, \omega\right) \times \mathbf{k}_{1} C_{m l, \mu^{\prime}}\left(\mathbf{q}, \mathbf{k}, \mathbf{k}^{\prime}, \omega, t\right)\right]\right\} \\
& \times u_{\mu}^{\dagger}(\mathbf{q}) u_{\mu^{\prime}}\left(\mathbf{q}-\mathbf{k}^{\prime}\right) \\
& \dot{C}_{e l, \mu}\left(\mathbf{q}, \mathbf{k}, \mathbf{k}^{\prime}, \omega, t\right)=\frac{1}{(2 \pi)^{3} \sqrt{\pi \varepsilon_{0} \hbar}} \sum_{\mu^{\prime}=1}^{2} \int d^{3} \mathbf{k}_{1} \frac{\omega}{c^{2}} e^{-i\left(\tilde{\omega}_{A}-\omega-\omega_{B}\right) t} C_{u, \mu^{\prime}}(\mathbf{q}, t) \\
& \times g_{e}^{*}\left(\mathbf{k}_{1}-\mathbf{k}, \omega\right) u_{\mu}^{\dagger}\left(\mathbf{q}-\mathbf{k}^{\prime}\right) u_{\mu^{\prime}}(\mathbf{q})\left\{\omega \mathbf{G}^{*}\left(-\mathbf{k}_{1},-\mathbf{k}^{\prime}, \omega\right)\right. \\
& \left.+\mathbf{G}^{*}\left(-\mathbf{k}_{1},-\mathbf{k}^{\prime}, \omega\right) \times \mathbf{k}^{\prime} \times \dot{\mathbf{R}}\right\} \cdot \hat{\mathbf{d}}_{A}, \\
& \dot{C}_{m l, \mu}\left(\mathbf{q}, \mathbf{k}, \mathbf{k}^{\prime}, \omega, t\right)=\frac{1}{(2 \pi)^{3} \sqrt{\pi \varepsilon_{0} \hbar}} \sum_{\mu^{\prime}=1}^{2} \int d^{3} \mathbf{k}_{1} \frac{1}{c} e^{-i\left(\tilde{\omega}_{A}-\omega-\omega_{B}\right) t} C_{u, \mu^{\prime}}(\mathbf{q}, t) \\
& \times g_{m}^{*}\left(\mathbf{k}_{1}-\mathbf{k}, \omega\right) u_{\mu}^{\dagger}\left(\mathbf{q}-\mathbf{k}^{\prime}\right) u_{\mu^{\prime}}(\mathbf{q}) \\
& \times\left\{i \omega \mathbf{k}_{1} \times \mathbf{G}^{*}\left(-\mathbf{k}_{1},-\mathbf{k}^{\prime}, \omega\right)\right. \\
& \left.+i \mathbf{k}_{1} \times \mathbf{G}^{*}\left(-\mathbf{k}_{1},-\mathbf{k}^{\prime}, \omega\right) \times \mathbf{k}^{\prime} \times \dot{\mathbf{R}}\right\} \cdot \hat{\mathbf{d}}_{A} .
\end{aligned}
$$

Suppose that the system is prepared in a state described by $|\mathbf{q}\rangle_{\mu}|u\rangle|\{0\}\rangle$. Therefore, at the initial time $t=0$, we have $C_{u, \mu}(\mathbf{q}, 0)=1$ (for $\mu=1$ or $\mu=2$ ) and $C_{\lambda l, \mu}\left(\mathbf{q}, \mathbf{k}, \mathbf{k}^{\prime}, \omega, 0\right)=0$. At any time $t>0$, we can calculate the amplitude probability that the moving atom has emitted a photon by 
substituting the solution of Eqs. (48) and (49) into (47) and using the integral relation (38) in the Fourier space. Then, after a lengthy calculation, we obtain

$$
\begin{aligned}
\dot{C}_{u}(\mathbf{q}, t)= & -i \delta \omega C_{u}(\mathbf{q}, t) \\
+ & \sum_{\mu, \mu^{\prime}=1}^{2} \int d^{3} \mathbf{k}^{\prime} u_{\mu}^{\dagger}(\mathbf{q}) u_{\mu^{\prime}}\left(\mathbf{q}-\mathbf{k}^{\prime}\right) u_{\mu^{\prime}}^{\dagger}\left(\mathbf{q}-\mathbf{k}^{\prime}\right) u_{\mu}(\mathbf{q}) \\
& \times \int_{0}^{t} d t^{\prime} \mathbb{K}\left(\mathbf{k}^{\prime}, t-t^{\prime}\right) C_{u}\left(\mathbf{q}, t^{\prime}\right),
\end{aligned}
$$

where we have used $\sum_{\mu} C_{u, \mu}(\mathbf{q}, t) u_{\mu}(\mathbf{q})=C_{u}(\mathbf{q}, t)$. Here, the kernel function $\mathbb{K}\left(\mathbf{k}^{\prime}, t-t^{\prime}\right)$ can be written in terms of the imaginary part of the Green tensor of the system as

$$
\begin{aligned}
\mathbb{K}\left(\mathbf{k}^{\prime}, t-t^{\prime}\right) & =-\frac{1}{(2 \pi)^{3}\left(\hbar \pi \varepsilon_{0}\right)} \int_{0}^{\infty} \frac{d \omega}{c^{2}} e^{-i\left(\tilde{\omega}_{A}-\omega-\omega_{B}\right)\left(t^{\prime}-t\right)} \\
& \times \hat{\mathbf{d}}_{A} \cdot\left\{\omega^{2} \operatorname{Im} \mathbf{G}\left(\mathbf{k}^{\prime}, \omega\right)+\omega \dot{\mathbf{R}} \times \mathbf{k}^{\prime} \times \operatorname{Im} \mathbf{G}\left(\mathbf{k}^{\prime}, \omega\right)\right. \\
& \left.+\omega \operatorname{Im} \mathbf{G}\left(\mathbf{k}^{\prime}, \omega\right) \times \mathbf{k}^{\prime} \times \dot{\mathbf{R}}+\dot{\mathbf{R}} \times \mathbf{k}^{\prime} \times \operatorname{Im} \mathbf{G}\left(\mathbf{k}^{\prime}, \omega\right) \times \mathbf{k}^{\prime} \times \dot{\mathbf{R}}\right\} \cdot \hat{\mathbf{d}}_{A},
\end{aligned}
$$

in which we have defined $\mathbf{G}\left(\mathbf{k}^{\prime}, \omega\right)=\mathbf{G}\left(\mathbf{k}^{\prime}, \mathbf{k}^{\prime}, \omega\right)$. To make further progress, in first step, it is necessary to compute $\sum_{\mu, \mu^{\prime}=1}^{2} u_{\mu}^{\dagger}(\mathbf{q}) u_{\mu^{\prime}}\left(\mathbf{q}-\mathbf{k}^{\prime}\right) u_{\mu^{\prime}}^{\dagger}\left(\mathbf{q}-\mathbf{k}^{\prime}\right) u_{\mu}(\mathbf{q})$. To do so, we can extend the sums over $\mu$ and $\mu^{\prime}$ to include all four values. We can do this by using

$$
\frac{c \boldsymbol{\alpha} \cdot \mathbf{q}+\beta M c^{2}+\left|E_{\mathbf{q}}\right|}{2\left|E_{\mathbf{q}}\right|} u_{\mu}(\mathbf{q})= \begin{cases}u_{\mu}(\mathbf{q}) & \text { for } \mu=1,2 \\ 0 & \text { for } \mu=3,4\end{cases}
$$

and a similar relation for $u_{\mu^{\prime}}\left(\mathbf{q}-\mathbf{k}^{\prime}\right)$. Now, we consider the completeness relation $\sum_{\mu=1}^{4} u_{\mu}\left(\mathbf{q}-\mathbf{k}^{\prime}\right) u_{\mu}^{\dagger}\left(\mathbf{q}-\mathbf{k}^{\prime}\right)=\mathbb{I}$, therefore, we arrive at

$$
\begin{aligned}
& \sum_{\mu, \mu^{\prime}=1}^{2} u_{\mu}^{\dagger}(\mathbf{q}) u_{\mu^{\prime}}\left(\mathbf{q}-\mathbf{k}^{\prime}\right) u_{\mu^{\prime}}^{\dagger}\left(\mathbf{q}-\mathbf{k}^{\prime}\right) u_{\mu}(\mathbf{q})=\frac{1}{4\left|E_{\mathbf{q}}\right|\left|E_{\mathbf{q}-\mathbf{k}^{\prime}}\right|} \\
& \times \operatorname{Tr}\left[\left(c \boldsymbol{\alpha} \cdot\left(\mathbf{q}-\mathbf{k}^{\prime}\right)+\beta M c^{2}+\left|E_{\mathbf{q}-\mathbf{k}^{\prime}}\right|\right)\left(c \boldsymbol{\alpha} \cdot \mathbf{q}+\beta M c^{2}+\left|E_{\mathbf{q}}\right|\right)\right] .
\end{aligned}
$$

The above trace can be evaluated using the identity $\operatorname{Tr}[(\boldsymbol{\alpha} \cdot \mathbf{a})(\boldsymbol{\alpha} \cdot \mathbf{b})]=4 \mathbf{a} \cdot \mathbf{b}$ and the fact that the trace of a product of any odd number of the matrices $\sigma_{x}, \sigma_{y}, \sigma_{z}$ and $\beta$ is zero. Therefore, Eq. (53) becomes

$$
1+\sqrt{\left(1-v_{1}^{2} / c^{2}\right)\left(1-v_{2}^{2} / c^{2}\right)}+\frac{\mathbf{v}_{\mathbf{1}} \cdot \mathbf{v}_{\mathbf{2}}}{c^{2}}
$$


where $\mathbf{v}_{\mathbf{1}}$ and $\mathbf{v}_{\mathbf{2}}$ are the velocities before and after the emission of the photon. If the momentum of the photon is negligible compared to the center-of-mass momentum of the atom, then $\mathbf{v}_{\mathbf{1}} \simeq \mathbf{v}_{\mathbf{2}}$ and the above relation become equal to two. This is true in both the classical limit and the extreme relativistic limit.

In the next step, to obtain an analytic solution for the probability amplitude (50), we restrict our attention to the case that the atom-field system coupled weakly. We use the Markov approximation and replace the coefficient $C_{u}\left(\mathbf{q}, t^{\prime}\right)$ by $C_{u}(\mathbf{q}, t)$, and let the upper integration limit in the above equation tend to infinity, then approximate the time integral $\int_{0}^{\infty} d t^{\prime} e^{-i\left(\tilde{\omega}_{A}-\omega-\omega_{B}\right)\left(t^{\prime}-t\right)} \approx$ $\zeta\left(\omega+\omega_{B}-\tilde{\omega}_{A}\right)$ by the zeta function $\zeta(x)=\pi \delta(x)+i P(1 / x)$, in which $P$ stands for Cauchy principal value.

Some remarks on the Markov approximation are now in order. First of all, it is interesting to note that there have been various theoretical studies and debates on the problem of the quantum friction force when an atom moves at constant velocity near a macroscopic body [35, 36, 37, 38, 39, 40, 41, 42, 43, 44, 45, 46]. The result is a velocity dependent force causes the atom to decelerate, but leads to contradictory predictions concerning its dependence on velocity and with atom-surface separation. Unfortunately, due to the short range and extremely small magnitude of this force, there is no experimental confirmation yet with presently available technology to judge which of theoretical results is correct. Therefore, we cannot decide whether any of these results is flawed. To clarify the origins of these disagreement between quantum friction calculations, Intravaia et al. [44, 45] showed that the Markov approximation fails to provide reliable predictions for both in equilibrium and out of equilibrium quantum friction. With this in view, Klatt et al. [46] based on Markovian quantum master equations and time-dependent perturbation theory have obtained interesting results concerning the quantum frictional force and the dynamical corrections to the level shifts and decay rates. It is observed that the two approaches agree for the level shifts, the decay rates, and the velocity and distance dependency of the quantum frictional force, if considering the second order in the atom-field coupling. In contrast, both approaches for the quantum friction force differ to the terms of fourth order in coupling [46].

With the above background and employing the above approximations, we arrive at the familiar result $C_{u, \mu}(\mathbf{q}, t)=\exp \left(-\frac{1}{2} \Gamma+i \delta \omega\right) t(\mu=1$ or $\mu=2)$, where $\Gamma$ and $\delta$ are, respectively, the decay rate of the excited atom and the Lamb shift in the laboratory frame. These parameters are defined in terms 
of the Green tensor of the system as:

$$
\begin{aligned}
\Gamma= & \frac{2}{(2 \pi)^{3} \hbar \varepsilon_{0} c^{2}} \int d^{3} \mathbf{k}^{\prime} \int d \omega \delta\left(\omega-\left(\frac{\omega_{0}}{\gamma}-\delta \omega\right)-\dot{\mathbf{R}} \cdot \mathbf{k}^{\prime}\right) \\
& {\left[\hat { \mathbf { d } } { } _ { A } \cdot \left\{\omega^{2} \operatorname{Im} \mathbf{G}\left(\mathbf{k}^{\prime}, \omega\right)+\omega \dot{\mathbf{R}} \times \mathbf{k}^{\prime} \times \operatorname{Im} \mathbf{G}\left(\mathbf{k}^{\prime}, \omega\right)\right.\right.} \\
& +\omega \operatorname{Im} \mathbf{G}\left(\mathbf{k}^{\prime}, \omega\right) \times \mathbf{k}^{\prime} \times\left(\dot{\mathbf{R}}-\frac{\hbar \mathbf{k}^{\prime}}{\gamma M}\right) \\
& \left.\left.+\dot{\mathbf{R}} \times \mathbf{k}^{\prime} \times \operatorname{Im} \mathbf{G}\left(\mathbf{k}^{\prime}, \omega\right) \times \mathbf{k}^{\prime} \times\left(\dot{\mathbf{R}}-\frac{\hbar \mathbf{k}^{\prime}}{\gamma M}\right)\right\} \cdot \hat{\mathbf{d}}_{A}\right], \\
\delta \omega= & \frac{1}{(2 \pi)^{3} \hbar \varepsilon_{0} \pi c^{2}} \int d^{3} \mathbf{k}^{\prime} P \int \frac{d \omega}{\omega-\left(\frac{\omega_{0}}{\gamma}-\delta \omega\right)-\dot{\mathbf{R}} \cdot \mathbf{k}^{\prime}} \\
& {\left[\hat { \mathbf { d } } \cdot \left\{\omega^{2} \operatorname{Im} \mathbf{G}\left(\mathbf{k}^{\prime}, \omega\right)+\omega \operatorname{Im} \mathbf{G}\left(\mathbf{k}^{\prime}, \omega\right) \times \mathbf{k}^{\prime} \times\left(\dot{\mathbf{R}}-\frac{\hbar \mathbf{k}^{\prime}}{\gamma M}\right)\right.\right.} \\
& +\omega \dot{\mathbf{R}} \times \mathbf{k}^{\prime} \times \operatorname{Im} \mathbf{G}^{\prime}\left(\mathbf{k}^{\prime}, \omega\right) \\
& \left.\left.+\dot{\mathbf{R}} \times \mathbf{k}^{\prime} \times \operatorname{Im} \mathbf{G}\left(\mathbf{k}^{\prime}, \omega\right) \times \mathbf{k}^{\prime} \times\left(\dot{\mathbf{R}}-\frac{\hbar \mathbf{k}^{\prime}}{\gamma M}\right)\right\} \cdot \hat{\mathbf{d}}_{A}\right] .
\end{aligned}
$$

Here, the center-of-mass velocity of the atom before and after the emission of photon are, respectively, given by $\dot{\mathbf{R}}=\hbar \mathbf{q} / M$ and $\dot{\mathbf{R}}-\hbar \mathbf{k}^{\prime} / \gamma M$. Obviously, it is seen that the decay rate $\Gamma$ and the frequency shift $\delta \omega$ have been affected by the atomic motion through complicated relations that can be calculated by having the Green's tensor of any arbitrary medium, but due to the complexity of relations, its analytical calculations are practically impossible and should be investigated numerically. Now, in the limiting case where the surrounding environment of the moving atom is vacuum, the Green tensor of the system is written as follows

$$
\operatorname{Im} \mathbf{G}_{\alpha \beta}(\mathbf{k}, \omega)=\left(\delta_{\alpha \beta}-k_{\alpha} k_{\beta}\right) \frac{\pi}{2 \omega c} \delta\left(|\mathbf{k}|-\frac{\omega}{c}\right) .
$$

By substituting the above equation into Eq. (55), as a well known result of special relativity, we find that $\Gamma=\frac{\omega_{0}^{3}\left|\mathbf{d}_{0 A}\right|^{2}}{3 \pi \varepsilon_{0} \hbar c^{3} \gamma}=\frac{\Gamma_{0}}{\gamma}$, wherein $\mathbf{d}_{0 A}$ and $\Gamma_{0}$ are, respectively, the transition dipole matrix element and the free space rate of decay of the atom in the rest frame of the atom. This agreement also provides an independent check of the theoretical method introduced in this article. 


\section{5. conclusions}

In this paper, a canonical quantization of the electromagnetic field interacting with moving charge particles is presented in the presence of an isotropic, homogeneous, and absorbing magnetodielectric medium. To achieve this purpose, an appropriate Lagrangian is introduced and the Hamiltonian of the combined system is derived. A unitary transformation has been applied on the Hamiltonian and the Hamiltonian in a multipolar form is obtained. The formalism is generalized to include the relativistic motion of a atom, and then the evolution of the atomic system is determined in Schrödinger picture. By using the Markov approximation and finding the probability amplitudes of the system state vector, we got a general relation for the spontaneous emission rate and the Lamb shift of a moving atom with relativistic velocities in the presence of dissipation magnetodielectric mediums. It is shown that the dissipation effects of the surrounding environment on the spontaneous emission rate have been entered in our calculations through the Green tensor of the system, while the relativistic motion effects are introduced via the complicated relations including the parameter $\gamma$.

\section{Reference}

\section{References}

[1] P.W. Milonni, The quantum vacuum: an introduction to quantum electrodynamics, Academic Press, 1994.

[2] W. Guo, Phy. Rev. A. 77 (2008) 062111.

[3] M. Wilkens, Phys. Rev. A. 49 (1994) 570.

[4] L.G. Boussiakou, C.R. Bennett, M. Babiker, Phys. Rev. Lett. 89 (2002) 123001.

[5] J.D. Cresser, S.M. Barnett, J. Phys. B: At. Mol. Opt. Phys. 36 (2003) 1755.

[6] E. Hinds, S.M. Barnett, Phys. Rev. Lett. 102 (2009) 050403.

[7] S.M. Barnett, R. Loudon, Proc. R. Soc. London, Ser. A. 368 (2010) 927.

[8] S.M. Barnett, Phys. Rev. Lett. 104 (2010) 070401. 
[9] P.W. Milonni and R.W. Boyd, Adv. Opt. Photon. 2 (2010) 519.

[10] U. Leonhardt, M. Wilkens, Europhys. Lett. 42 (1998) 365.

[11] U. Leonhardt, P. Piwnicki, Phys. Rev. Lett. 82 (1999) 2426.

[12] S. Horsley, M. Babiker, Phys. Rev. Lett. 95 (2005) 010405.

[13] A. Ashkin, Optical trapping and manipulation of neutral particles using lasers: a reprint volume with commentaries, World Scientific, 2006.

[14] S. Scheel, S.Y. Buhmann, Phy. Rev. A. 80 (2009) 042902.

[15] G. Barton, New J. Phys. 12 (2010) 113045.

[16] S. Lannebre, M.G. Silveirinha, J. Opt. 19 (2017) 014004.

[17] F. Kheirandish, E. Amoghorban, Phy. Rev. A. 82 (2010) 042901.

[18] E. Amooghorban, M. Wubs, N.A. Mortensen, F. Kheirandish, Phys. Rev. A. 84 (2011) 013806.

[19] M.M. Behbahani, E. Amooghorban, A. Mahdifar, Phys. Rev. A. 94 (2016) 013854.

[20] B. Huttner, S.M. Barnett, Phys. Rev. A. 46 (1992) 4306.

[21] J. Jeffers, S.M. Barnett, R. Loudon, R. Matloob, M. Artoni, Opt Commun. 131 (1996) 66.

[22] L.G. Suttorp, M. Wubs, Phys. Rev. A. 70 (2004) 013816.

[23] M. Amooshahi, J. Math. Phys 50 (2009) 062301.

[24] T.G. Philbin, New J. Phys. 12 (2010) 123008.

[25] J.J. Hopfield, Phy. Rev. 112 (1996) 1555.

[26] H.T. Dung, S.Y. Buhmann, L. Knöll, D.-G. Welsch, J. Kästel, Phys. Rev. A. 68 (2003) 043816.

[27] S.Y. Buhmann, D.-G. Welsch, Prog. Quantum Electron. 31 (2007) 51. 
[28] V.E. Lembessis, M. Babiker, C. Baxter, R. London, Phys. Rev. A. 48 (1993) 1594.

[29] J.C. Guilot, J. Robert, J. Phys. A: Math. Gen. 35 (2002) 5023.

[30] H.A. Bethe, E.E. Salpeter, Quantum mechanics of one- and two-electron atoms, Springer Science and Business Media, 1957.

[31] S.Y. Buhmann, Dispersion Forces I, Springer, 2013.

[32] S.Y. Buhmann, Dispersion Forces II, Springer, 2013.

[33] E. Amoghorban, F. Kheirandish, Internat. J. Theoret. Phys. 53 (2014) 2593.

[34] N.N. Bogoliubov, D.V.E. Shirkov, S. Chomet, Introduction to the Theory of Quantized Fields, John Wiley and Sons, New York, 1980.

[35] J. B. Pendry, J. Phys.: Condens. Matter 9 (1997) 10301.

[36] A.I. Volokitin and B.N.J. Persson, Phys. Rev. B 74 (2006) 205413.

[37] T.G. Philbin and U. Leonhardt, New J. Phys. 11 (2009) 033035.

[38] J.B. Pendry, New J. Phys. 12 (2010) 033028.

[39] G. Barton, New J. Phys. 12 (2010) 113044.

[40] G. Barton, New J. Phys. 12 (2010) 113045.

[41] G.V. Dedkov, and A.A. Kyasov, Phys. Solid State 44 (2002) 1809.

[42] V.A. Golyk, M. Kruger and M. Kardar, Phys. Rev. B 88 (2013) 155117.

[43] G. Pieplow and C. Henkel, New J. Phys. 15 (2013) 023027.

[44] F. Intravaia, V.E. Mkrtchian, S.Y. Buhmann, S. Scheel, D.A.R. Dalvit, C. Henkel, J. Phys.: Condens. Matter. 27 (2015) 214020.

[45] F. Intravaia, R.O. Behunin, C. Henkel, K. Busch, D.A.R. Dalvit, Phys. Rev. A. 94 (2016) 042114.

[46] J. Klatt, M.B. Faras, D.A.R. Dalvit, S.Y. Buhmann, Phys. Rev. A. 95 (2017) 052510. 